$\begin{gathered}\text { Advanced Research in } \\ \text { Gastroenterology \& Hepatology } \\ \text { ISSN: 2472-6400 }\end{gathered}$
$\begin{aligned} & \text { Mini Review } \\ & \text { Volume 12 Issue 1 - January 2019 } \\ & \text { Dol: 10.19080/ARGH.2019.12.555829 }\end{aligned}$
Copyright $\odot$ All rights are reserved by Karen J Campoverde Reyes

\title{
Noninvasive Evaluations of Non-alcoholic Fatty Liver Disease in Pediatric Populations
}

\author{
Karen J Campoverde Reyes ${ }^{1,2 *}$, Aysha Aslam ${ }^{1}$ and Pir Ahmad Shah ${ }^{1}$ \\ ${ }^{1}$ Division of Gastroenterology, Beth Israel Deaconess Medical Center, Harvard Medical School, Boston, United States of America \\ ${ }^{2}$ Neuroendocrine Unit, Massachusetts General Hospital, Harvard Medical School, Boston, United States of America
}

Submission: December 12, 2018; Published: January 17, 2019

*Corresponding author: Karen J Campoverde Reyes, Liver Center, BIDMC, Harvard Medical School, 110 Francis Street, Suite 4A, Boston, MA 02115, USA

Keywords: NAFLD; Pediatrics; Steatosis; Fatty liver; Diagnostic tools; Liver disease

Abbreviations: NAFLD: Nonalcoholic Fatty Liver Disease; NASH: Nonalcoholic Steatohepatitis; IHTG: Intrahepatic Triglycerides; HCC: Hepatocellular Carcinoma; MRE: Magnetic Resonance Elastography; VLDL: Very Low-Density Lipoprotein

\section{Mini Review}

Nonalcoholic fatty liver disease (NAFLD) is the most common liver disease in the pediatric population Schwimmer JB et al. [1], Anderson et al. [2] \& Le et al. [3]. NAFLD in children is associated with central obesity and insulin resistance Alterio \& Vos et al. $[4,5]$. The prevalence of NAFLD in children with obesity is estimated to be $34.2 \%$. That is significantly higher than the $7.6 \%$ among children from the general population Anderson et al. [2]. The understanding of the pathogenesis of NAFLD is essential to ensure the finding of a reproducible and accurate noninvasive tool to effectively detect the progress of hepatic steatosis Loomba [6]. This mini review focuses on the currently available noninvasive diagnostic modalities that can potentially be applied in children populations.

The natural history of NAFLD progresses from steatosis to nonalcoholic steatohepatitis (NASH) to cirrhosis. The term NASH is specifically associated with inflammation and subsequent fibrosis compared to simple steatosis. The stage of liver fibrosis has been identified as the strongest predictor of overall mortality and liver disease complications in adults Angulo et al. [7]. NAFLD-related cirrhosis is currently the second leading cause for liver transplantation in adults Charlton et al. [8]. The risk of hepatocellular carcinoma (HCC) increases with cirrhosis; however, recent report suggests that HCC can also develop in non-cirrhotic NAFLD Welzel et al. [9]. Patients with NAFLD are at higher risk of having other complications such as type 2 diabetes mellitus, cardiovascular diseases, and cancer compared to the general population Lonardo et al. [10]. Similarly, children with NAFLD also were noted to have increased cardiovascular risk and systemic health complications Baskar [11]. The natural history of NAFLD in pediatric populations has not been well established due to the paucity of prospective longitudinal studies. Available cross-sectional studies suggested that the entire spectrum of NALFD also occur in children. Nobili et al. [12] However, the prognosis and clinical complications of NAFLD in pediatric populations need to be carefully evaluated by prospective studies.

Simple steatosis, or fatty liver, occurs early in NAFLD and may not be associated with elevated liver enzymes. Moreover, up to $23 \%$ of children with NAFLD can have a normal ALT even with the presence of liver fibrosis. Schwimmer, et al. [13], Mencin [14], Schwimmer, et al. [15] \& Temple et al. [16]. It is estimated that $25 \%$ of pediatric patients with NAFLD will progress to NASH. In a study on pediatric NAFLD patients, 5 had serial liver biopsies over 5 years. Four of them were noted to have progressive advanced fibrosis Feldstein AE et al. [17].

Liver biopsy is considered the gold standard for diagnosing and evaluating the severity of NAFLD. It is, however, an expensive invasive procedure associated with sampling error and potential serious complications. Its acceptance in the pediatric population is, therefore, limited Kalia et al. [18] \& Le et al. [3].

Noninvasive modalities to monitor disease progression in pediatric patients with NAFLD are greatly needed. Abdominal ultrasound is readily available but has limited sensitivity. It can only detect moderate to severe fatty infiltration of the liver $(\geq$ $30 \%$ steatosis). Furthermore, it is operator-dependent and cannot quantify fat and fibrosis Bouchi, et al. [19], Jiang ZG, et al. [20] \& Mencin [14]. Despite their limitations, abdominal ultrasound and serum aminotransferases remain the first choice in screening NAFLD in children Temple et al. [16]. Magnetic resonance elastography (MRE) can quantify steatosis and fibrosis with high accuracy and reliability in patients with NAFLD but it 
has limited availability and is not cost-effective as a screening modality Temple, et al. [16] \& Dulai et al. [21]. Transient elastography (Fibro-scan) is another relatively new noninvasive method for the assessment of liver steatosis and fibrosis. It is fast with generally accurate and reproducible results. It is, however, less reliable in pediatric patients with obesity and is not available in most community hospitals in USA Francavilla [22].

Pathogenesis of NAFLD is complex and is directly associated with the metabolism of lipoproteins. The lipolysis of subcutaneous adipose tissue and intrahepatic and intraabdominal fat leads to an increase in fatty acids, with an increase in intrahepatic triglycerides (IHTG) content. That subsequently leads to elevated production of VLDL and triglycerides. Fatty acid overload in the hepatocytes results in an impairment of the insulin signal pathway causing decreased insulin action on glycogen synthase with subsequent insulin resistance increase in glucose secretion. Hepatocytes attempt to dispose the excessive triglyceride accumulation in the liver by increasing the VLDL secretion. However, the generation of free fatty acids and VLDL is not balanced, it is actually ineffective and results in inflammation and accumulation of fat in the liver. With the increasing fat deposition in the liver, the processing of intrahepatic fats via various lipoproteins such as low density -LDL, very low densityVLDL, high density-HDL is also altered, resulting in change in the serum concentration of these molecules Nobili et al. [12] Fabbrini et al. [23], Jiang ZG et al. [24], Lavine et al. [25] \& Perla et al. [26].

Lipoprotein analysis by Nuclear Magnetic Resonance (NMR) is an assay that provides lipoprotein information based on the lipid methyl signals. A given size particle has a constant number of terminal lipid methyl groups and each lipoprotein particle of a particular size has its own NMR signature. NMR spectrum is analyzed to provide particle concentrations for each of the VLDL, LDL and HDL subclasses. By size VLDL particles, for example, can be divided as large (>60 nm), medium ( $42-60 \mathrm{~nm})$, and small (29-42 nm). Likewise, LDL particles can separate into large (20.5-23 nm) and small (18-20.5 nm) particles; HDL particles are classified into 3 categories by size: large (9.4-14 nm), medium (8.2-9.4nm) and small (7.3-8.2 nm). Some of the advantages of using NMR detection include negligible sample preparation, unbiased detection and its inherently quantitative without the need for control standard.

In adults, it has been shown that the progression of NAFLD is accompanied by distinctive changes in very low-density lipoprotein (VLDL) Jiang ZG et al. [24], Garcia et al. [27] \& Amor et al. [28]. In a single center study of adult patients with NAFLD, an increase in VLDL particle size was noted to be association with both NAFLD activity score (NAS) and NASH. In addition, a decrease in small VLDL particle concentration was associated with more advanced liver fibrosis Jiang ZG et al., [24]. Hence, this NMR profile of lipoprotein particles can potentially be used as a reliable method to detect progression of NAFLD in the future.
Its availability along with its noninvasive nature could hold a special interest in the pediatric population. These interesting and important observations need to be further validated in both adult and pediatric populations before it can be applied to clinical practice. It is imperative to find noninvasive and costeffective tools that can accurately monitor progression of NAFLD in the clinical settings of pediatric populations [29-31].

\section{References}

1. Schwimmer JB, Deutsch R, Kahen T, Lavine JE, Stanley C, et al. (2006) Prevalence of fatty liver in children and adolescents. Pediatrics 118(4): 1388-1393.

2. Anderson EL, Howe LD, Jones HE, Higgins JP, Lawlor DA, et al. (2015) The prevalence of non-alcoholic fatty liver disease in children and adolescents: a systematic review and meta-analysis. PloS one 10(10): e0140908.

3. Le MH, Devaki P, Ha NB, Jun DW, Te HS (2017) Prevalence of non-alcoholic fatty liver disease and risk factors for advanced fibrosis and mortality in the United States. PloS one 12(3): e0173499.

4. Alterio A, Alisi A, Liccardo D, Nobili V (2014) Non-alcoholic fatty liver and metabolic syndrome in children: a vicious circle. Horm Res Paediatr 82(5): 283-289.

5. Vos MB, Abrams SH, Barlow SE, Caprio S, Daniels SR, et al. (2017) NASPGHAN clinical practice guideline for the diagnosis and treatment of nonalcoholic fatty liver disease in children: recommendations from the Expert Committee on NAFLD (ECON) and the North American Society of Pediatric Gastroenterology, Hepatology and Nutrition (NASPGHAN). J Pediatr Gastroenterol Nutr 64(2): 319-334.

6. Loomba R (2017) Role of imaging-based biomarkers in NAFLD: Recent advances in clinical application and future research directions. J Hepatol 68(2): 296-304.

7. Angulo P, Kleiner DE, Dam-Larsen S, Adams LA, Bjornsson ES, et al. (2005). Liver fibrosis, but no other histologic features, is associated with long-term outcomes of patients with nonalcoholic fatty liver disease. Gastroenterology 149(2): 389-397.

8. Charlton MR, Burns JM, Pedersen RA, Watt KD, Heimbach JK (2011) Frequency and outcomes of liver transplantation for nonalcoholic steatohepatitis in the United States. Gasteroenterology 41(4): 1249-1253.

9. Welzel TM, Graubard BI, Quraishi S, Zeuzem S, Davila JA, et al. (2013) Population-attributable fractions of risk factors for hepatocellular carcinoma in the United States. Cancer 122(11): 1757-1765.

10. Lonardo A, Sookoian S, Chonchol M, Loria P, Targher G (2013) Cardiovascular and systemic risk in nonalcoholic fatty liver disease-atherosclerosis as a major player in the natural course of NAFLD. Curr Pharm Des 19(29): 5177-5192.

11. Baskar SJ (2015) Cardiovascular risk in pediatric nonalcoholic fatty liver disease: recent advances. Clinical Lipidology 351-362.

12. Nobili V, Svegliati-Baroni G, Alisi A, Miele L, Valenti L (2013) A 360-degree overview of paediatric NAFLD: Recent insights. J Hepatol 58(6):1218-1229.

13. Schwimmer JB, Celedon MA, Lavine JE, Salem R, Campbell N, et al. (2009) Heritability of nonalcoholic fatty liver disease. Gastroenterology 136(5): 1585-1592.

14. Mencin AA (2011) Advances in pediatric nonalcoholic fatty liver disease. Pediatric Clinics 1375-1392.

15. Schwimmer JB, Newton KP, Awai HI, Choi LJ, Garcia MA, et al. (2013) Paediatric gastroenterology evaluation of overweight and obese children referred from primary care for suspected non-alcoholic fatty liver disease. Aliment Pharmacol Ther 38(10): 1267-1277. 
16. Temple JL, Cordero P, Li J, Nguyen V, Oben JA (2016) A guide to non-alcoholic fatty liver disease in childhood and adolescence. Int J Mol Sci $17(6)$.

17. Feldstein AE, Charatcharoenwitthaya P, Treeprasertsuk S, Benson JT, Enders FB, et al. (2009) The natural history of non-alcoholic fatty liver disease in children: a follow-up study for up for 20 years. Gut 58(11): 1538-1544.

18. Kalia HS, Gaglio PJ (2016) The prevalence and pathobiology of nonalcoholic fatty liver disease in patients of different races or ethnicities. Clin Liver Dis 20(2): 215-224

19. Bouchi R, Takeuchi T, Akihisa M, Ohara N, Nakano Y, et al. (2016) Increased visceral adiposity with normal weight is associated with the prevalence of non-alcoholic fatty liver disease in Japanese patients with type 2 diabetes. J Diabetes Investig 7(4): 607-614

20. Jiang ZG, Robson SC, Yao Z (2013) Lipoprotein metabolism in nonalcoholic fatty liver disease. J Biomed Res 27(1): 1-13.

21. Dulai PS, Sirlin CB, Loomba R (2016) MRI and MRE for non-invasive quantitative assessment of hepatic steatosis and fibrosis in NAFLD and NASH: Clinical trials to clinical practice. J Hepatol 65(6): 1006-1016.

22. Francavilla R (2009) C010 Normal values of transient elastography (fibroscan) in children without evidence of liver disease: comparison of adult versus pediatric probe. Digestive and Liver disease.

23. Fabbrini E, Mohammed BS, Magkos F, Korenblat KM, Patterson BW, et al. (2008) Alterations in adipose tissue and hepatic lipid kinetics in obese men and women with nonalcoholic fatty liver disease. Gastroenterology 134(2): 424-431.
24. Jiang ZG, Tapper EB, Connelly MA, Pimentel CF, Feldbrügge L (2016) Steatohepatitis and liver fibrosis are predicted by the characteristics of very low density lipoprotein in nonalcoholic fatty liver disease. Liver Int 36(8): 1213-1220.

25. Lavine JE, Schwimmer JB, Molleston JP, Scheimann AO, Murray KF (2010) Treatment of nonalcoholic fatty liver disease in children: TONIC trial design. Contemp Clin Trials 31(1): 62-70.

26. Perla FM, Prelati M, Lavorato M, Visicchio D, Anania C (2017) The Role of Lipid and Lipoprotein Metabolism in Non-Alcoholic Fatty Liver Disease. Children (Basel) 4(6).

27. Garcia AE, Kasim N, Tamboli RA, Gonzalez RS, Antoun J (2015) Lipoprotein profiles in class III obese caucasian and African American women with nonalcoholic fatty liver disease. PloS one 10(11): e0142676.

28. Amor AJ, Pinyol M, Solà E, Catalan M, Cofán M (2017) Relationship between noninvasive scores of nonalcoholic fatty liver disease and nuclear magnetic resonance lipoprotein abnormalities: A focus on atherogenic dyslipidemia. J Clin Lipidol 11(2): 551-561.

29. Chalasani N, Deeg MA, Crabb DW (2004) Systemic levels of lipid peroxidation and its metabolic and dietary correlates in patients with nonalcoholic steatohepatitis. Am J Gastroenterol 99(8): 1497-1582.

30. Marcason W (2013) What are the current guidelines for pediatric non-alcoholic fatty liver disease? J Acad Nutr Diet 113(12): 1772

31. Vilar-Gomez E, Chalasani N (2017) Non-invasive assessment of non-alcoholic fatty liver disease: Clinical prediction rules and blood-based biomarkers. J Hepatol 68(2): 305-315.

\section{Your next submission with JuniperPublishers will reach you the below assets}

- Quality Editorial service

- Swift Peer Review

- Reprints availability

- E-prints Service

- Manuscript Podcast for convenient understanding

- Global attainment for your research

- Manuscript accessibility in different formats

( Pdf, E-pub, Full Text, audio)

- Unceasing customer service

Track the below URL for one-step submission https://juniperpublishers.com/online-submission.php 